\title{
Alanine and arginine rich domain containing protein, Aard, is directly regulated by androgen receptor in mouse Sertoli cells
}

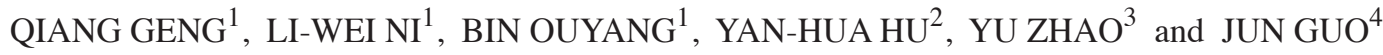 \\ ${ }^{1}$ First Teaching Hospital of Tianjin University of Traditional Chinese Medicine, Tianjin 300193; \\ ${ }^{2}$ Union Stem Cell \& Gene Engineering Co., Ltd, Tianjin 300384; \\ ${ }^{3}$ Graduate School of Tianjin University of Traditional Chinese Medicine, Tianjin 300193; \\ ${ }^{4}$ Department of Andrology, Xiyuan Hospital of China Academy of Chinese Medical Science,
}

Beijing 100091, P.R. China

Received September 17,2015; Accepted September 28, 2016

DOI: $10.3892 / \mathrm{mmr} .2016 .6028$

\begin{abstract}
Alanine and arginine rich domain containing protein (Aard) is specifically expressed in Sertoli cells (SCs) of mouse testis and the expression increases in an age-dependent manner. A number of previous studies have indicated that androgen and androgen receptor (AR) signaling pathways are particularly important for spermatogenesis in mouse SCs, however, the association between Aard and AR remain to be elucidated. The present study identified Aard as a gene that is directly regulated by AR in mouse SCs, which is important in spermatogenesis. The expression of AARD was significantly downregulated in the testes of Sertoli cell-selective AR knockout mice compared with wild-type mice as analyzed by western blotting and immunofluorescence analyses. Quantitative polymerase chain reaction and western blotting indicated that AARD was predominantly expressed in adult mouse testis and its expression was increased in an age-dependent manner. In addition, AARD expression was upregulated by testosterone in primary SCs in vitro, which was confirmed by bioinformatics analysis and a dual-luciferase reporter assay. Finally, chromatin immunoprecipitation and electrophoretic mobility shift assays indicated that the ligand-bound AR activated Aard transcription via directly binding to the androgen-responsive element of the Aard promoter. To the best of our knowledge, the present study is the first to document that Aard is directly regulated by AR in mouse Sertoli cells.
\end{abstract}

\section{Introduction}

Androgen and the androgen receptor (AR) are essential in male spermatogenesis and fertility (1-3). AR mediates physiological

Correspondence to: Professor Jun Guo, Department of Andrology, Xiyuan Hospital of China Academy of Chinese Medical Science, 1 Xiyuan Caochang, Haidian District, Beijing 100091, P.R. China E-mail: guojun1126@126.com

Key words: Aard, androgen receptor, testis, SCs and pathophysiological effects of androgens by binding to genomic androgen response elements (AREs) of target genes $(4,5)$. In human, mutations in the $A R$ gene can result in male infertility and cancer, including androgen insensitivity and testicular feminization syndrome, breast carcinoma and prostate cancer (6-8). Similarly, constitutive $A R$ knockout mice exhibit complete androgen insensitivity with testes that are larger and located at the abdomen, and their germ-cell development is severely disrupted (9).

Previous studies have demonstrated that germ cells do not express AR, and the effect of androgens on spermatogenesis is predominantly mediated via Sertoli cells (SCs). Sertoli cell-selective AR knockout (SCARKO) mice exhibit a complete disruption of meiosis, which demonstrates that SCs are important in regulating spermatogenesis (10-12). Although gene expression profile analysis demonstrated that a number of genes were regulated by androgens in mouse SCs, only a few genes have been identified as candidate target genes for AR, including ubiquitin conjugating enzyme E2 B, heat shock factor protein 1, reproductive homeobox 5 (Rhox5) and tubulin $\beta 3$ class III (Tubb3) (13-16). However, AR function during spermatogenesis is still not well understood. Further research is essential to determine which target genes are physiologically relevant and may be useful as diagnostic or therapy targets to modulate the effects of ARs in spermatogenesis.

The mouse alanine and arginine rich domain containing protein (Aard) gene was first identified as a gene exhibiting sexually dimorphic expression in fetal gonads using polymerase chain reaction (PCR)-based cDNA subtraction analysis. A previous study demonstrated that AARD was specifically expressed at higher levels in the testes of the developing mouse embryo relative to the ovaries and other tissues. Strong testis-specific expression of AARD was also detected in the adult mouse (17). Furthermore, AARD is exclusively located in SCs of XY gonads during sex differentiation (17). Notably, a previous study using digital gene expression analysis data also demonstrated that the Aard gene was markedly downregulated in SCARKO mouse testes compared with wild-type (WT) mice (18). This suggests that Aard may be a critical target gene of AR involved in spermatogenesis. 
The present study identified the AARD was specifically expressed in Sertoli cells (SCs) of the mouse testis and increased in an age-dependent manner, and its expression was markedly downregulated in SCARKO mouse testes. The chromatin immunoprecipitation and electrophoretic mobility shift assays demonstrated that Aard was a novel direct target gene of AR in mouse SCs. These observations suggest that the low expression of Aard in the testes of SCARKO mouse testes may be one of the factors that impair spermatogenesis and result in male infertility.

\section{Materials and methods}

Animals. C57BL/6 $(\mathrm{n}=30)$ and SCARKO $(\mathrm{n}=30)$ mice $(8$ weeks old) were obtained from the Model Animal Research Center of Nanjing University (Nanjing, China). Mice were housed in a pathogen-free environment at $\sim 22^{\circ} \mathrm{C}$ under a $12 \mathrm{~h}$ light/dark cycle. All the animals had free access to standard water and chow. All mice were treated according to the National Institutes of Health Guide for the Care and Use of Laboratory Animals. The study was approved by the ethics committee of Xiyuan Hospital of China Academy of Chinese Medical Science (Beijing, China).

Primary SCs cultures and transfection. Primary SCs were separated from 19-day-old mouse testes as previously described (19). Cells were cultured at $37^{\circ} \mathrm{C}$ in a $5 \% \mathrm{CO}_{2}$ humidified atmosphere with Dulbecco's modified Eagle's medium (Invitrogen; Thermo Fisher Scientific, Inc., Waltham, MA, USA) and 10\% fetal bovine serum (Invitrogen; Thermo Fisher Scientific, Inc.), and supplemented with $100 \mathrm{U} / \mathrm{ml}$ penicillin and $100 \mathrm{U} / \mathrm{ml}$ streptomycin (Invitrogen; Thermo Fisher Scientific, Inc.).

Primary SCs were seeded in a 6 or 24-well plate and transfected using Lipofectamine 2000 (Invitrogen; Thermo Fisher Scientific, Inc.) according to the manufacturer's protocols. $A R$ siRNA (siAr) was obtained from Shanghai GenePharma, Co., Ltd. (Shanghai, China). The sequences were as follows: 5'-AGGACUUGCUGUCAUUGAAAUGGA-3' for siAR; and 5'-CAGCAUAUUAGAAUAGCGCGACA-3' for siNC. $\mathrm{Ar}$ overexpression plasmid (pCDNA4.1-AR) and corresponding empty vector (pCDNA4.1) were synthesized by Thermo Fisher Scientific, Inc.

Quantitative PCR ( $q P C R)$. RNA was extracted from mouse tissues using TRIzol (Invitrogen; Thermo Fisher Scientific, Inc.). Total RNA extraction and $\mathrm{qPCR}$ reactions were performed as previously described (20) using the following kits: Reverse transcription, ReverPrimeScript RT Enzyme Mix I kit (Takara Bio, Inc., Otsu, Japan); qPCR, SYBR ${ }^{\circledR}$ Premix

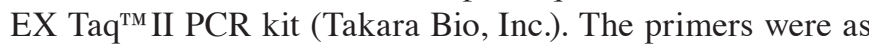
follows: Sense, 5'-AGAGCCCGCAGGATAAGGAGAT-3' and antisense, 5'-AGTGTTAATGCTAGGAGGGTTTCA-3' for mouse Aard; sense, 5'-CTCTTTCAAGGGAGGTTACGCC-3' and antisense, 5'-CTGGTATGCTGCTGCCTCGTCT-3' for $A R$; and sense, 5'-TTCCAGCCTTCCTTCTTGGGTAT-3' and antisense, 5'-GTTGGCATAGAGGTCTTTACGG-3' for Actb, which served as an internal control. The relative expression levels of Aard and $A R$ were evaluated according to the $2^{-\Delta \Delta C q}$ method (21).
Western blotting. Protein extracts of different mouse tissues and primary SCs using radioimmunoprecipitation assay buffer (Invitrogen; Thermo Fisher Scientific, Inc.). The protein concentration was quantified using a bicinchoninic acid assay kit (Pierce; Thermo Fisher Scientific, Inc.). Samples $(20 \mu \mathrm{g})$ were subjected to $12 \%$ sodium dodecyl sulfate polyacrylamide gel electrophoresis and transferred to polyvinylidene fluoride membranes (EMD Millipore, Billerica, MA, USA). Following blocking with $5 \%$ non-fat milk in Tris-buffered saline with Tween 20, the membrane was then incubated with anti-AARD antibody (1:500; Santa Cruz Biotechnology, Dallas, TX, USA; cat. no. sc-86960), anti-AR antibody (1:1,000; Abcam, Cambridge, UK; cat. no. ab133273), and anti-ACTB (1:10,000; Abcam; cat. no. ab8226) overnight at $4{ }^{\circ} \mathrm{C}$, followed by horseradish peroxidase (HRP)-conjugated corresponding secondary antibody (1:2,000; Abcam; cat. no. ab97051) for $1 \mathrm{~h}$ at $37^{\circ} \mathrm{C}$. A chemiluminescence phototube-HRP kit (EMD Millipore, Billerica, MA, USA) was used to visualize the immunoreactive bands.

Immunofluorescence double staining. Adult mice were sacrificed using an intraperitoneal injection of $3 \%$ pentobarbital sodium $(50 \mathrm{mg} / \mathrm{kg})$ and the testes were fixed in $4 \%$ paraformaldehyde solution for $48 \mathrm{~h}$ at $37^{\circ} \mathrm{C}$. Tissues were processed into paraffin using standard techniques and sectioned at 3-4 $\mu \mathrm{m}$ thickness. Following blocking in $10 \%$ bovine serum albumin (Beyotime Institute of Biotechnology, Haimen, China), the sections were then incubated with the goat anti-AARD antibody (1:200) and rabbit anti-transcription factor SOX-9 (SOX9; 1:300; Abcam; cat. no. ab185230) antibody overnight at $4^{\circ} \mathrm{C}$. Sections were subsequently washed with phosphate-buffered saline and performed with appropriate fluorescein isothiocyanate- or tetramethylrhodamine-conjugated secondary antibodies (1:500; Abcam; cat. nos. ab6717 and ab6718, respectively) for $1 \mathrm{~h}$ at $37^{\circ} \mathrm{C}$. Finally, the sections were stained with Hoechst 33342 (1 mg/ml; Invitrogen; Thermo Fisher Scientific, Inc.). Representative sections were photographed using a laser scanning confocal microscope (Zeiss AG, Oberkochen, Germany) and images were analyzed using the Coreldraw 9 (Corel Corporation, Ottawa, Canada).

Plasmid construction and dual-luciferase reporter assay. Bioinformatics analysis was performed in order to identify the potential ARE region in the Aard promoter. The promoter sequences of Aard were obtained using the National Center for Biotechnology Information (www.ncbi.nlm.nih.gov/) and the UCSC Genome bioinformatics brwoser (genome.ucsc.edu/). Subsequently, an ARE structure (TGTTCT) at position 1637 to 1632 in the region extending from -2000 bp relative to the Aard transcription initiation site was identified. Luciferase reporter plasmid assay was performed using the psiCHECK-2 as a basic vector. The Aard promoter (WT) was sub-cloned into psiCHECK-2 vector by KpnI and NheI sites. The primers for the Aard promoter was as follows: Forward, 5'-CGGGGT ACCAGGTATGAGCTCCACTCAGTATTT-3' and reverse, 5'-CTAGCTAGCGCGGGGGCAGTTAACGGAACAGGC A-3'. The mutant Aard promoter (MUT) has the same sequence as the WT Aard promoter excluding the mutation at position -1637 to -1632 (TGTTCT to CACCTC) was conducted using the QuickChange II site-directed mutagenesis kit (Stratagene; 
Agilent Technologies, Inc., Santa Clara, CA, USA) with appropriate primers according to the manufacturer's instructions. All recombinant plasmids were verified by DNA sequencing performed by Invitrogen (Thermo Fisher Scientific, Inc).

Primary SCs were seeded in a 24 -well plate at $\sim 4 \times 10^{4}$ cells/well, and transiently co-transfected with pcDNA4.1-AR or pcDNA4.1 empty vector together with recombinant psiCHECK-2 WT or MUT reporter plasmids using Lipofectamine 2000. After 6-8 h transfection, cells were treated with $10 \mathrm{nM}$ testosterone or ethanol vehicle for $24 \mathrm{~h}$. Firefly luciferase and Renilla activities were detected using the dual luciferase reporter system (Promega Corporation, Madison, WI, USA) with a Modulus ${ }^{\mathrm{TM}}$ Single Tube Multimode Reader (Bio-Systems International, Beloit, WI, USA). Firefly luciferase activity was used to normalize Renilla luciferase activity. Following normalization for transfection efficiency, induction factors were analyzed as the ratios of the mean value of the luciferase signal in the testosterone-stimulated samples compared with untreated samples. The mouse mammary tumor virus long terminal repeat served as a positive control.

Electrophoretic mobility shift assay (EMSA). EMSA was conducted using a Light-Shift Chemiluminescent EMSA kit (Thermo Fisher Scientific, Inc.), according to the manufacturer's protocols. Primary SCs were cultured in 100-mm dishes and transiently transfected with pcDNA4.1-AR or pcDNA4.1 empty vector for $24 \mathrm{~h}$ using Lipofectamine 2000. Subsequently, cells were treated with $10 \mathrm{nM}$ testosterone for $24 \mathrm{~h}$ prior to the EMSA. The WT probe 5'-CACCCC TGCCCCTGTTCTGTGTGCACACGT-3' and MUT probe 5'-CACCCCTGCCCCCACCTCGTGTGCACACGT-3' from the mouse Aard promoter, including the potential ARE structure (in bold), were obtained from Thermo Fisher Scientific, Inc., and treated using Biotin 3' End DNA Labeling kit (Pierce; Thermo Fisher Scientific, Inc.). Binding reactions were performed at $37^{\circ} \mathrm{C}$ for $20 \mathrm{~min}$ in the binding buffer according to the manufacturer's protocols. Another 50- or 200 -fold molar excess of the unlabeled WT probe was used for the competitive assay. Subsequently, the AR antibody ( $5 \mu \mathrm{l} ; 1: 200)$ was added for AR super-shift analysis and incubated for $2 \mathrm{~h}$ at $37^{\circ} \mathrm{C}$. Finally, the DNA-protein complexes were separated in a $6 \%$ non-denaturing polyacrylamide gel by electrophoresis, transferred to nylon membranes and detected using enhanced chemiluminescence detection kit (Pierce; Thermo Fisher Scientific, Inc.) and a FluorChem M imaging system (ProteinSimple, San Jose, CA, USA).

Chromatin immunoprecipitation (ChIP) assay. Primary $\mathrm{SCs}$ were treated as described above for the EMSA assay. Samples without testosterone treatment were regarded as the negative control. The detailed procedure for extraction of chromatin from cells and subsequent chromatin immunoprecipitation reaction was performed as previously (22). Immunoprecipitated DNA fragments were analyzed by semi-quantitative PCR amplification using primers for Aard. The primers for the Aard ARE bind region were as follows: Sense, 5'-CAGGTGCCAGCACTACAGAACCAGT-3' and antisense, 5'-ACTGGTTCTGTAGTGCTGGCA CCTG-3'. The AR target genes, Rhox5 and Tubb3, served as positive controls. The primers for were as follows: Forward, 5'-GGAGGGCAACACCAGTCCCTG-3' and reverse, 5'-CTCGGTGTCGCAAAAGGGCA-3' for Rhox5 ARE bind region; and forward, 5'-TGGCCCCCAGAACAGAAG-3' and reverse, 5'-TGGTGTTCCCACTCTGTACAATG-3' for Tubb3. The PCR was performed using EmeraldAmp PCR Master Mix kit (Takara Bio, Inc.) and the following cycling conditions: $98^{\circ} \mathrm{C}$ for $2 \mathrm{~min} ; 32$ cycles of $98^{\circ} \mathrm{C}$ for $10 \mathrm{sec}$, $60^{\circ} \mathrm{C}$ for $30 \mathrm{sec}$ and $72^{\circ} \mathrm{C}$ for $30 \mathrm{sec}$; and $72^{\circ} \mathrm{C}$ for $5 \mathrm{~min}$. The amplified products were separated on a $2 \%$ agarose gel and visualized with ultraviolet imaging system (Bio-Rad Laboratories, Inc., Hercules, CA, USA).

Statistical analysis. Each experiment was repeated at least three times. Data were expressed as the mean \pm standard error of the mean. Statistical analysis was analyzed using SSPS 16.0 (SPSS, Inc., Chicago, IL, USA). Student's t-test was used to compare the difference between different groups. $\mathrm{P}<0.05$ was considered to indicate a statistically significant difference.

\section{Results}

Expression of AARD in SCARKO and WT mouse testes. Previous microarray analysis data indicated that AARD was downregulated in SCARKO mouse testis (18). Thus, AARD expression was first demonstrated in adult SCARKO and WT mouse testes using western blot and immunofluorescence analysis. Consistent with the expectations of the present study, the results demonstrated that AARD was significantly decreased in the testes of adult SCARKO mice compared with WT mice $(\mathrm{P}<0.05$; Fig. 1A). As presented in Fig. 1B, AARD protein was also downregulated in SCARKO mouse testes. AARD was exclusively located in the nucleus of SCs (Fig. 1C), which was verified by co-immunostaining with SOX9, an approved SCs marker.

Temporal expression pattern of AARD during mouse testis development. To investigate the function and underlying molecular mechanisms of AARD during mouse testis development, qPCR and western blotting were performed to evaluate the distribution of AARD expression in different adult mouse tissues. The results demonstrated that Aard mRNA and protein was predominantly expressed in mouse testis (Fig. 2A). The temporal expression of AARD was further analyzed during postnatal testis development at mRNA and protein levels. As presented in the Fig. 2B, the data indicated that AARD is expressed in the testes during all stages and increased from the postnatal 1 week to 8 weeks, which suggested Aard may be important during the process of spermatogenesis.

Activation of AARD expression induced by testosterone in $S C s$ in vitro. To investigate whether testosterone can promote AARD expression, mediated via AR, primary SCs were transiently transfected with a pcDNA4.1-AR overexpression plasmid and treated with $10 \mathrm{nM}$ testosterone for $24 \mathrm{~h}$. The results indicated that AARD expression was significantly upregulated at the mRNA level $(\mathrm{P}<0.05)$ and markedly upregulated at the protein level, as compared with testosterone untreated samples (Fig. 3A). However, the mRNA expression level of AARD was significantly downregulated $(\mathrm{P}<0.05)$ and 
A

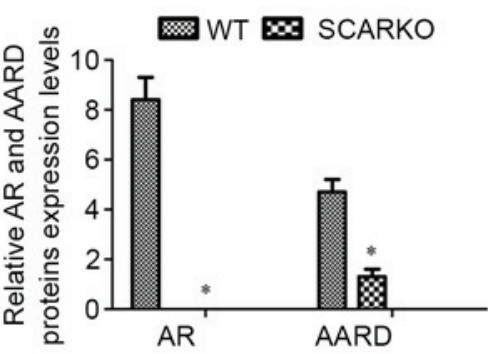

B

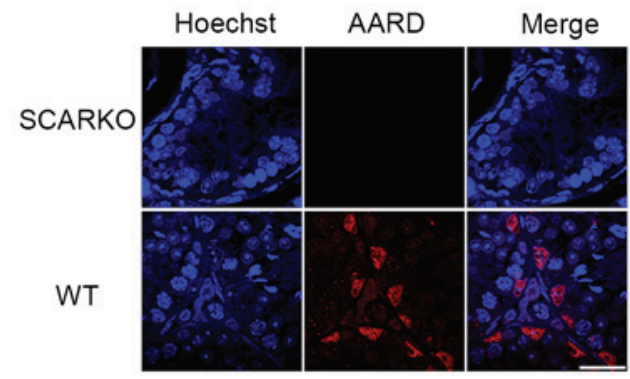

C

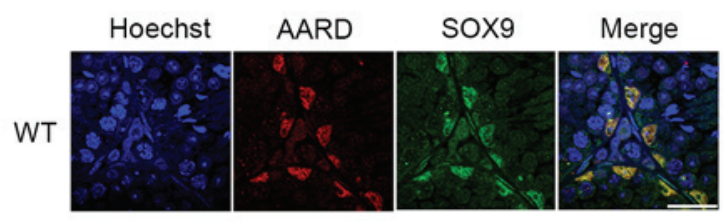

Figure 1. Expression levels of AARD in SCARKO and WT mouse testes. (A) The expression of AARD in adult SCARKO and WT mouse testes were detected by western blot analysis. (B) Immunofluorescence assay was also used to examine AARD expression in adult SCARKO and WT mouse testes. "P $<0.05$ vs. WT group. (C) Localization of AARD, as assessed by anti-AARD antibody (green) and a Sertoli cell marker SOX9 (red). Cell nuclei were labeled with Hoechst (blue). The protein expression level of AARD was markedly downregulated in SCARKO mice compared with WT mouse. Scale bar=20 $\mu \mathrm{m}$. AARD, alanine and arginine rich domain containing protein; AR, androgen receptor; SCARKO, Sertoli cell-selective AR knockout; WT, wild-type; ACTB, $\beta$-actin; SOX-9, transcription factor SOX-9.

A

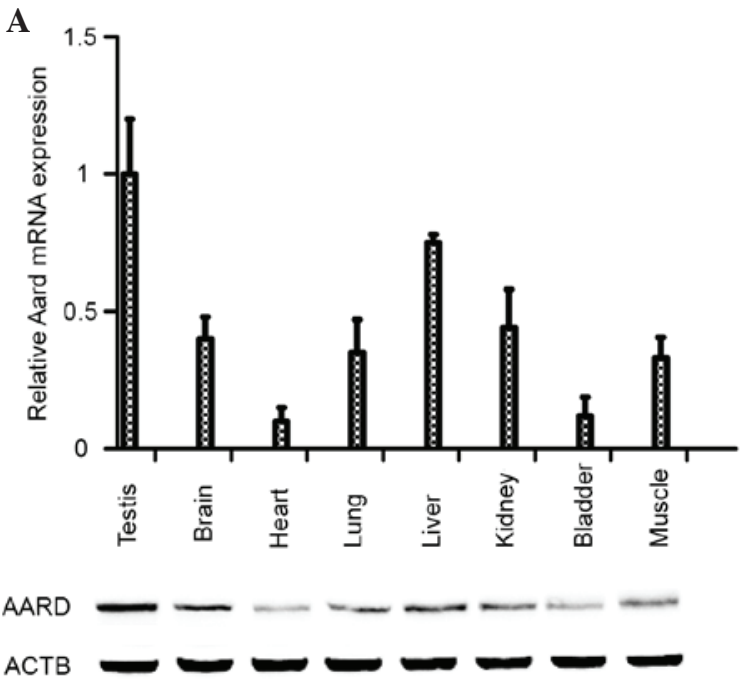

B

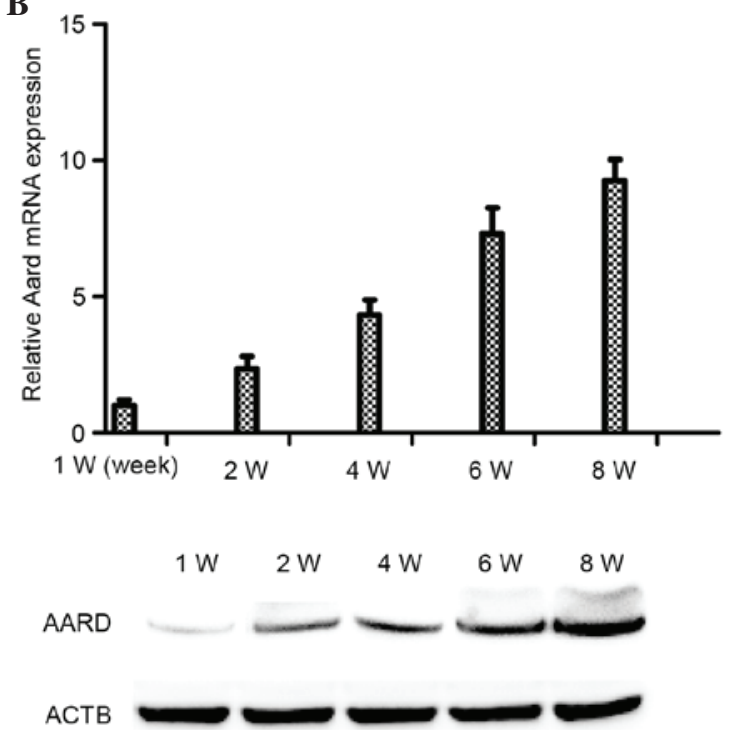

Figure 2. Temporal expression pattern of AARD during mouse testis development. (A) The mRNA and protein expression levels of AARD in different tissues of adult WT mice were detected by qPCR and western blotting. Following normalization to ACTB, the relative expression level of Aard in different tissues was compared with that in the testis. Data are expressed as the mean \pm standard error of the mean $(n=5)$. (B) The age-dependent expression of AARD in mouse testes. qPCR and western blotting were used to determine the expression of AARD in mouse testes at indicated times. AARD, alanine and arginine rich domain containing protein; ACTB, $\beta$-actin; WT, wild-type; qPCR, quantitative polymerase chain reaction.

the protein level notably downregulated following treatment of SCs with siAr oligonucleotides (Fig. 3B). These results strongly indicated that AARD expression was activated by testosterone and mediated via AR. 
A
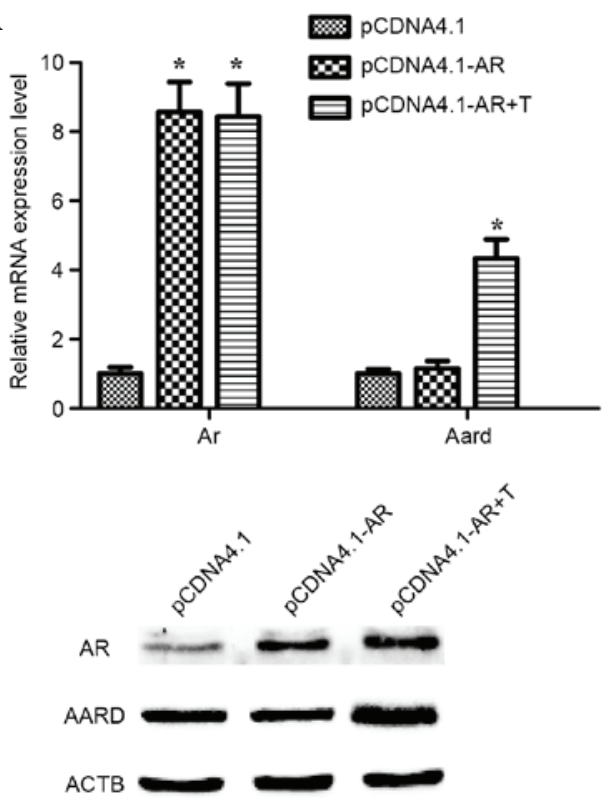

B

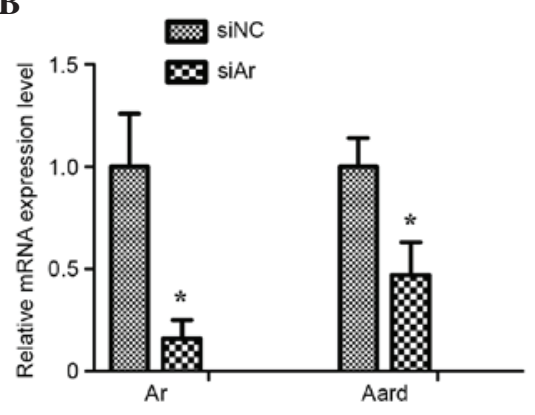

Figure 3. Activation of AARD expression induced by testosterone in SCs in vitro. (A) SCs were transfected with an AR expression plasmid and treated with $10 \mathrm{nM}$ testosterone for $24 \mathrm{~h}$. The expression of AARD in SCs was examined by quantitative polymerase chain reaction and western blotting. $* \mathrm{P}<0.05$ vs.pCDNA4.1.(B) The expression level of AARD was significantly downregulated following treatment of SCs with siAr oligonucleotides. Data were expressed as the mean \pm standard error of the mean, ${ }^{*} \mathrm{P}<0.05$ vs. siNC. T, testosterone; SCs, Sertoli cells; AR, androgen receptor; AARD, alanine and arginine rich domain containing protein; siNC, negative control small interfering RNA; siAR, AR small interfering RNA; ACTB, $\beta$-actin.

A

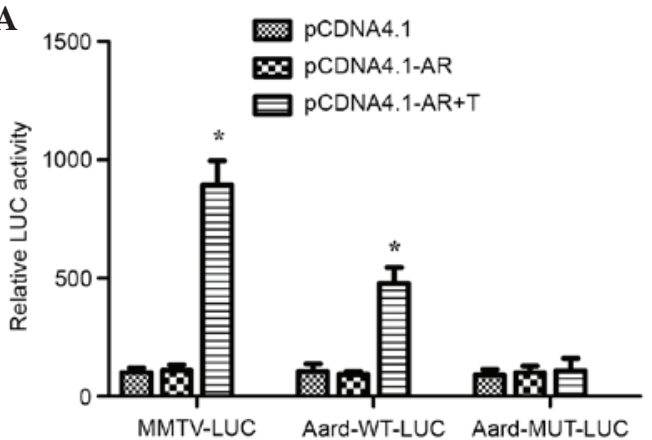

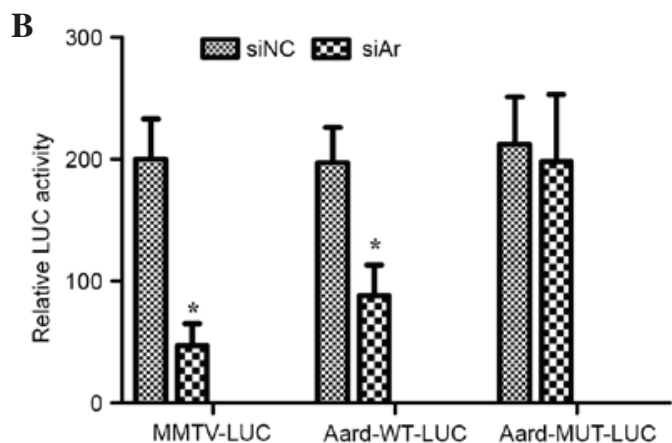

Figure 4. Promotion of Aard promoter activity by testosterone in SCs. A dual-luciferase reporter assay was used to detect the activation of Aard promoter activity by testosterone in SCs cells. Positive control samples were performed with the testosterone-inducible MMTV recombination vector. (A) SCs were transfected with an AR expression plasmid and treated with $10 \mathrm{nM}$ testosterone for $24 \mathrm{~h}$. Results were presented as fold-change of testosterone-treated samples relative to testosterone-untreated samples. " $\mathrm{P}<0.05$ vs. pcDNA4.1-AR. (B) The luciferase activity was evaluated following treatment of SCs with siAr oligonucleotides. ${ }^{*} \mathrm{P}<0.05$ vs. siNC. Data were presented as the mean \pm standard deviation. LUC, luciferase; WT, wild-type; MUT, mutant; T, testosterone; SCs, Sertoli cells; AR, androgen receptor; AARD, alanine and arginine rich domain containing protein; siNC, negative control small interfering RNA; siAR, AR small interfering RNA; MMTV, mouse mammary tumor virus long terminal repeat.

Promotion of Aard promoter activity by testosterone in SCs. In order to assess the function of testosterone in the activation of the transcription of Aard, a dual-luciferase reporter assay was performed. Primary SCs were transfected with WT or MUT Aard promoter luciferase plasmids, and then were conducted with $10 \mathrm{nM}$ testosterone. Notably, it was observed that the relative luciferase activity of WT Aard promoter was significantly promoted by testosterone $(\mathrm{P}<0.05)$, while sequences change from TGTTCT $(-1637$ to -1632) to CACCTC in the ARE blocked Aard promoter regulation by testosterone in SCs (Fig. 4). Collectively, these results suggest that testosterone activated Aard, which is mediated via the AR by directly binding to the ARE in the Aard promoter.
Directly binding of $A R$ to the ARE in Aard promoter. To determine whether AR could directly bind to the ARE in Aard promoter, EMSA and ChIP assays were performed (Fig. 5A). A putative ARE was observed in the Aard promoter using bioinformatics analysis. As presented in Fig. 5B, lane 1 was used as a negative control, the nuclear extracts of AR overexpressing SCs treated with $10 \mathrm{nM}$ testosterone could notably bind to the WT ARE probe (lane 2). In addition, this binding ability was fully inhibited by addition of 50- or 200-fold molar excess of unlabeled ARE probe (cold probe; lane 3). By contrast, the binding was not eliminated by addition of the MUT ARE probe (lane 4). Furthermore, AR was identified in the ARE-Aard/AR complex by supershift analysis with an anti-AR antibody (lane 5). 
A

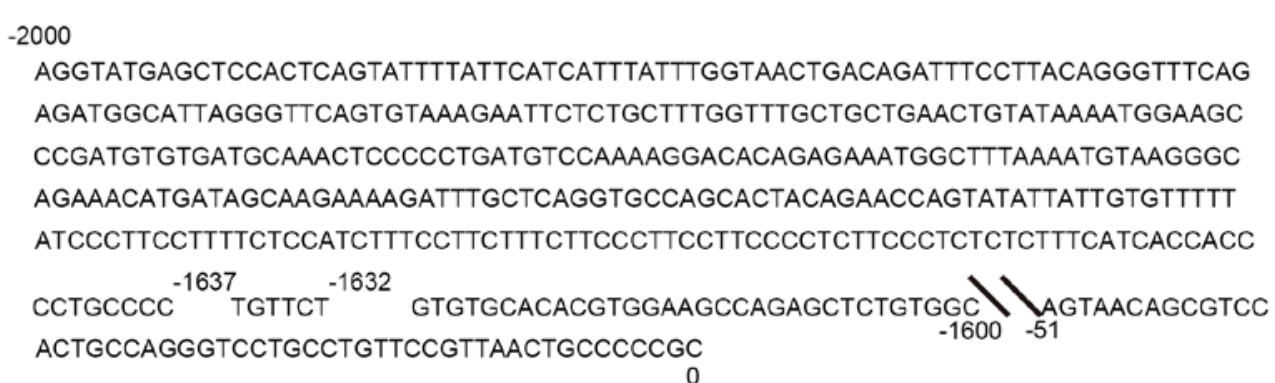

B

$\begin{array}{cc}\text { WT probe } & \text { 5'- СACCCCTGCCCCTGTTCTGTGTGCACACGT -3' } \\ \text { MUT probe } & \text { 5'- CACCCCTGCCCCCACCTCGTGTGCACACGT-3' }\end{array}$
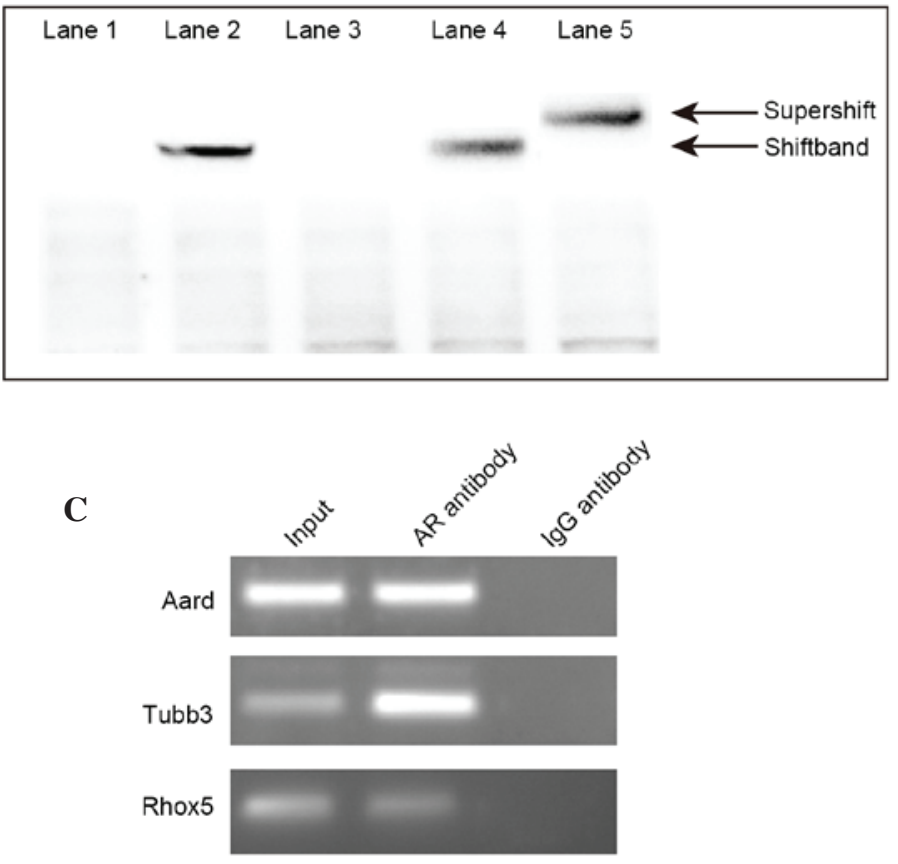

Figure 5. Direct binding of AR to the ARE in Aard promoter. (A) Sequences of putative ARE in the Aard promoter. (B) Electrophoretic mobility shift assay competition experiments. Lane 1, the mobility of the labeled WT probe without nuclear extracts; lane 2, the mobile ability of the labeled WT probe with nuclear extracts; lane 3, sample treated with 50- or 200-fold molar excess of unlabeled ARE-like probe (cold probe); lane 4, a similar excess of MUT probe, which the AR does not bind with; lane 5, super-shift assays with WT probe and nuclear extracts in the presence of anti-AR antibody. (C) Chromatin immunoprecipitation analysis demonstrated that AR could directly bind to the Aard promoter ARE in SCs in the presence of testosterone. Polymerase chain reaction amplification of eluted DNA was performed using specific primers for the Aard promoter region. WT, wide type; MUT, mutant; AR, androgen receptor; ARE, androgen-responsive element; IgG, immunoglobulin G; Aard, alanine and arginine rich domain containing protein; Tubb3, tubulin $\beta 3$ class III; Rhox5, reproductive homeobox 5 .

A ChIP assay was also conducted to assess the binding capacity of the AR to the ARE in the Aard promoter. SCs were transiently transfected with pcDNA4.1-AR overexpression plasmid and then treated with $10 \mathrm{nM}$ testosterone. AR binding to the ARE of the Aard promoter (-1637 to-1632) was confirmed by semi-quantitative PCR amplification using primers for Aard in anti-AR antibody-treated samples compared with anti-IgG antibody (Fig. 5B). These results indicate that AR has the ability to directly bind the Aard promoter DNA sequence.

\section{Discussion}

Normal spermatogenesis requires the action of androgen and the $\operatorname{AR}(1,23,24)$. Although androgens are sufficient to drive spermatogenesis, the underlying molecular mechanisms require further elucidation. The present study identified Aard as a novel target of the AR in mouse SCs. The present study demonstrated that the AR upregulated Aard expression by directly binding to its promoter, which may provide information regarding the molecular mechanism for abnormal spermatogenesis and male infertility.

Aard is specifically located in mouse SCs. The presence of a predicted leucine-zipper domain and a phosphorylation site in the AARD protein domain suggested that AARD may be involved in transcriptional regulation or intracellular signal transduction pathways (25). Based on analysis of SCARKO and WT mouse testes, the present study demonstrated that AARD had significantly reduced expression in SCARKO mouse testes compared with WT mouse. These functions of androgen on AARD expression were also observed following restoration 
or knockdown expression of $A R$ in primary SCs-treated with testosterone in vitro. These data demonstrated that AR-regulated Aard expression was critical for spermatogenesis.

To distinguish between the possibilities that the effect of androgens involved a direct interaction of the AR with the Aard gene or was indirectly mediated via an androgen-regulated intermediary transcription factor, functional AREs were searched for in the promoter region extending from -2000 bp relative to the Aard transcription initiation site. Previous studies demonstrated that the canonical ARE binding site is composed of a dimer to an inverted repeat segregated by three oligonucleotides (AGAACAnnnTGTTCT). The atypical AREs contain the half-site ARE sequence TGTTCT or the inverted complement AGAACA; head-to-head AREs [AGAACA $(0-8 \mathrm{n})$ TGTTCT, $\mathrm{n} \neq 3$ ]; tail-to-tail AREs [TGTTCT $(0-8 \mathrm{n})$ AGAACA]; or ARE direct repeats [AGAACA (0-8 n) AGAACA] $(26,27)$. Based on the above principles and combined with the matrix-scan tool of the regulatory sequences analysis tools software package (rsat.ulb.ac.be/rsat/), a potential ARE sequence (-1637 to-1632, TGTTCT) was observed to be located in the Aard promoter. This ARE binds to AR in vitro and is crucial for androgen-dependent reporter expression, based on the dual-luciferase reporter assay. Furthermore, EMSA and ChIP assays further proved that AR can directly bind to DNA in the Aard promoter.

In conclusion, the results of the present study indicate that Aard is a target of AR action in mouse SCs and suggest a novel finding by which the loss of AR function in SCs blocks spermatogenesis and results in male infertility. In normal mouse SCs, AR promotes AARD expression by directly binding to its promoter region. The loss of AR function resulted in a decrease in AARD expression in SCARKO mice. The relatively low expression in SCARKO mice may impair normal spermatogenesis, leading to male infertility. These results support a role for the AR-AARD axis in spermatogenesis, and further implicate the AR-AARD axis as a potential therapeutic target, particularly for those with male infertility resulting from aberrations in AR expression.

\section{References}

1. Heemers HV and Tindall DJ: Androgen receptor (AR) coregulators: A diversity of functions converging on and regulating the AR transcriptional complex. Endocr Rev 28: 778-808, 2007.

2. Patrão MT, Silva EJ and Avellar MC: Androgens and the male reproductive tract: An overview of classical roles and current perspectives. Arq Bras Endocrinol Metabol 53: 934-945, 2009.

3. Lazaros L, Xita N, Takenaka A, Sofikitis N, Makrydimas G, Stefos T, Kosmas I, Zikopoulos K, Hatzi E and Georgiou I: Semen quality is influenced by androgen receptor and aromatase gene synergism. Hum Reprod 27: 3385-3392, 2012.

4. Heinlein CA and Chang C: Androgen receptor (AR) coregulators: An overview. Endocr Rev 23: 175-200, 2002.

5. Nantermet PV, Xu J, Yu Y, Hodor P, Holder D, Adamski S, Gentile MA, Kimmel DB, Harada S, Gerhold D, et al: Identification of genetic pathways activated by the androgen receptor during the induction of proliferation in the ventral prostate gland. J Biol Chem 279: 1310-1322, 2004

6. Marcelli M, Ittmann M, Mariani S, Sutherland R, Nigam R, Murthy L, Zhao Y, DiConcini D, Puxeddu E, Esen A, et al: Androgen receptor mutations in prostate cancer. Cancer Res 60: 944-949, 2000

7. Chen MJ, Vu BM, Axelrad M, Dietrich JE, Gargollo P, Gunn S, Macias CG, McCullough LB, Roth DR, Sutton VR and Karaviti LP: Androgen Insensitivity Syndrome: Management Considerations from Infancy to Adulthood. Pediatr Endocrinol Rev 12: 373-387, 2015.
8. Kim Y, Jae E and Yoon M: Influence of Androgen Receptor Expression on the Survival Outcomes in Breast Cancer: A Meta-Analysis. J Breast Cancer 18: 134-142, 2015.

9. Yeh S, Tsai MY, Xu Q, Mu XM, Lardy H, Huang KE, Lin H, Yeh SD, Altuwaijri S, Zhou X, et al: Generation and characterization of androgen receptor knockout (ARKO) mice: An in vivo model for the study of androgen functions in selective tissues. Proc Natl Acad Sci USA 99: 13498-13503, 2002.

10. De Gendt K, Swinnen JV, Saunders PT, Schoonjans L, Dewerchin M, Devos A, Tan K, Atanassova N, Claessens F, Lécureuil C, et al: A Sertoli cell-selective knockout of the androgen receptor causes spermatogenic arrest in meiosis. Proc Natl Acad Sci USA 101: 1327-1332, 2004.

11. Wang RS, Yeh S, Tzeng CR and Chang C: Androgen receptor roles in spermatogenesis and fertility: Lessons from testicular cell-specific androgen receptor knockout mice. Endocr Rev 30: 119-132, 2009.

12. Chang C, Lee SO, Wang RS, Yeh S and Chang TM: Androgen receptor (AR) physiological roles in male and female reproductive systems: Lessons learned from AR-knockout mice lacking AR in selective cells. Biol Reprod 89: 21, 2013.

13. Mou L, Zhang Q, Wang Y, Zhang Q, Sun L, Li C, Huang W, Yuan Y, Duan Y, Diao R, et al: Identification of Ube2b as a novel target of androgen receptor in mouse sertoli cells. Biol Reprod 89: 32, 2013.

14. Yang L, Wang Y, Zhang Q, Lai Y, Li C, Zhang Q, Huang W, Duan Y, Jiang Z, Li X, et al: Identification of Hsf 1 as a novel androgen receptor-regulated gene in mouse Sertoli cells. Mol Reprod Dev 81: 514-523, 2014.

15. Bhardwaj A, Rao MK, Kaur R, Buttigieg MR and Wilkinson MF: GATA factors and androgen receptor collaborate to transcriptionally activate the Rhox 5 homeobox gene in Sertoli cells. Mol Cell Biol 28: 2138-2153, 2008.

16. De Gendt K, Denolet E, Willems A, Daniels VW, Clinckemalie L, Denayer S, Wilkinson MF, Claessens F, Swinnen JV and Verhoeven G: Expression of Tubb3, a beta-tubulin isotype, is regulated by androgens in mouse and rat Sertoli cells. Biol Reprod 85: 934-945, 2011.

17. Svingen T, Beverdam A, Verma P, Wilhelm D and Koopman P: Aard is specifically up-regulated in Sertoli cells during mouse testis differentiation. Int J Dev Biol 51: 255-258, 2007.

18. Zhang QX, Zhang XY, Zhang ZM, Lu W, Liu L, Li G, Cai ZM, Gui YT and Chang C: Identification of testosterone-/androgen receptor-regulated genes in mouse Sertoli cells. Asian J Androl 14: 294-300, 2012.

19. Verhoeven G and Cailleau J: Testicular peritubular cells secrete a protein under androgen control that inhibits induction of aromatase activity in Sertoli cells. Endocrinology 123: 2100-2110, 1988.

20. Lutfalla G and Uze G: Performing quantitative reverse-transcribed polymerase chain reaction experiments. Methods Enzymol 410: 386-400, 2006.

21. Kim JM, Hwang SH, Song EJ, Lee SY, Kim YD, Lee CH, Lee MK, Chang CL and Lee EY: Comparative quantification of plasma hnRNP B1 mRNA in non-small cell lung cancer patients by real-time PCR. Korean J Lab Med 29: 249-255, 2009 (In Korean).

22. Fujii $\mathrm{H}$ and Fujita $\mathrm{T}$ : Isolation of Specific Genomic Regions and Identification of Their Associated Molecules by Engineered DNA-Binding Molecule-Mediated Chromatin Immunoprecipitation (enChIP) Using the CRISPR System and TAL Proteins. Int J Mol Sci 16: 21802-21812, 2015.

23. Smith LB and Walker WH: The regulation of spermatogenesis by androgens. Semin Cell Dev Biol 30: 2-13, 2014.

24. Walker WH: Testosterone signaling and the regulation of spermatogenesis. Spermatogenesis 1: 116-120, 2011.

25. Blomberg LA, Chan WY, Clerch LB and Massaro D: Molecular cloning and characterization of a novel gene upregulated early during postnatal rat lung development. Biochim Biophys Acta 1574: 391-398, 2002.

26. Wang Q, Li W, Liu XS, Carroll JS, Jänne OA, Keeton EK, Chinnaiyan AM, Pienta KJ and Brown M: A hierarchical network of transcription factors governs androgen receptor-dependent prostate cancer growth. Mol Cell 27: 380-392, 2007.

27. Spillane M, Schwarz N and Willoughby DS: Upper-body resistance exercise augments vastus lateralis androgen receptor-DNA binding and canonical Wnt/ $\beta$-catenin signaling compared to lower-body resistance exercise in resistance-trained men without an acute increase in serum testosterone. Steroids 98: 63-71, 2015. 